\title{
Vibration Data Analysis for a Commercial Aircraft*
}

\author{
T. McKelvey*, T. Abrahamsson** and L. Ljung* \\ *Dept. of EE, Linköping University, S-581 83 Linköping, Sweden \\ ${ }^{* *}$ Saab Military Aircraft, S-581 88 Linkping, Sweden
}

March 6, 1995

\section{Submitted to Automatica \\ Report LiTH-ISY-R-1773, ISSN 1400-3902}

\begin{abstract}
Using data from extensive vibrational tests of the new aircraft Saab 2000 a combined method for vibration analysis is studied. The method is based on a realization algorithm followed by standard prediction error methods (PEM). We find that the realization algorithm gives good initial model parameter estimates that can be further improved by the use of PEM. We use the method to get insights into the vibrational eigenmodes.
\end{abstract}

Keywords: System identification, modal analysis, state-space methods, realization algorithm, model validation, application.

\section{Introduction}

System Identification concerns the problem of building mathematical models from observed input-output data. This area formally includes a large number of industrial problems. Traditionally several of these have not been dealt with using conventional System Identification methodology. Vibrational analysis is one such problem area, where other approaches have been dominating, primarily direct Fourier domain analysis and realization based techniques.

Vibrational mode analysis is of interest in many industrial branches. A vibrational mode is characterized by frequency and damping and is directly related to the mechanical structure. Therefore mode monitoring can be of significant importance for failure detection, e.g. in turbines or oil platforms $[1,2]$. In the aircraft industry vibrational modes are estimated and analyzed both for safety and comfort purposes. The modes of the wing structure,

${ }^{*}$ This work was supported by the Swedish Research Council for Engineering Sciences (TFR), which is gratefully acknowledged. 
e.g., are of great importance to evaluate risks of oscillation and fatigue and for deciding forces at landing. A great deal of effort is therfore spent on establishing the vibrational structure modes of an aircraft, both by ground experiment and during experimental flight tests.

In this contribution we shall study data from vibrational (ground) experiment on the new commercial aircraft SAAB 2000, developed by SAAB Aircraft Company, Linköping, Sweden. We investigate a combination of methods based on realization algorithm and based on maximum-likelihood (prediction error) approaches. Parameterization issues and evaluation measures play an important role in the application, and these questions will be dealt with in some detail.

The paper is organized as follows. Section 2 deals with the basic problem formulation, while the chosen identification method (a combination of two approaches) is described in Section 3. In Section 4 some criteria for model validation are discussed. The experiments and results of the identification are in section 5. The final Section 6 contains the conclusions.

\section{Problem Formulation}

\subsection{Motivation}

Analyses made during the design process of an aircraft are regularly required to be substantiated by tests. The vibrational properties, used for e.g. flutter and landing load analyses, are usually validated by comparison with data from ground vibration tests. Also flight tests are performed in order to validate analytical model properties, such as vibrational damping versus speed. Traditionally, ground vibration tests are performed using an aircraft placed on a very flexible supporting mechanism. The excitation is made by a few, say up to ten, shakers limited to a few hundred Newtons in load magnitude. The test results reported in this paper is from an experimental study, in which the structural damping properties at extremely high vibrational magnitudes were of major concern. The vibrations were of such magnitude that traditional excitation was not feasible. Instead, the aircraft structure (see Figure 1) was tied down, by use of tensioned wires, and instantaneously let loose to vibrate in a free decaying motion. Using the methods presented

here, reliable results were quickly produced. Also the methods were found to be sufficiently robust for industrial use.

\subsection{Modal Parameters in Vibrational Analysis}

The acceleration at each point $y_{i}(t), i=1, \ldots p$, of a free-decaying subcritically damped linear vibrating structure can be described as a sum of exponentially damped sinusoidals, each with a natural frequency $\omega_{k}$ and a 
damping ratio $\zeta_{k}$

$$
y_{i}(t)=\sum_{k=1}^{n / 2} \beta_{i, k} e^{-\zeta_{k} \Omega_{k} t} \sin \left(\omega_{k} t+\alpha_{i, k}\right)
$$

where $\alpha_{i, k}$ and $\beta_{i, k}$ are real constants and $\Omega_{k}=\omega_{k} / \sqrt{1-\zeta_{k}^{2}}$.

The structural deformation pattern (amplitude and phase) related to each sinusoidal is referred to as the modal shapes. The frequencies and corresponding damping ratios and the modal shapes are altogether referred to as modal parameters of the structure.

Sampled measurements of the acceleration at a discrete set of locations of a vibrating mechanical structure can be modeled by the impulse response of a discrete time linear multi-output state-space model

$$
\begin{aligned}
x(t+1) & =A x(t)+B u(t), \\
y(t) & =C x(t)
\end{aligned}
$$

where $y(t) \in \mathbb{R}^{p}, u(t) \in \mathbb{R}$ and $x(t) \in \mathbb{R}^{n}$ with $n$ equal the order of the system. In (2) we let the time $t$ be normalized with the sampling period. The $p$ output channels of the model $y(t) \in \mathbb{R}^{p}$ describes the acceleration at $p$ points of the structure. Assuming all modes are sub-critically damped each complex conjugate eigenvalue pair of the $A$ matrix in the linear state-space model (2) is associated with a complex conjugate pair of vibrating modes. From this eigenvalue pair the frequency and damping ratio can easily be determined.

Denote by $f$ the sampling frequency and by $\lambda_{k}^{+}(A), k=1, \ldots, n / 2$, the eigenvalues of $A$ from the state-space model with positive imaginary part. The frequencies and damping ratios are then determined by

$$
\begin{gathered}
\omega_{k}=\operatorname{Im}\left(\log \lambda_{k}^{+}\right) f \\
\Omega_{k}=\left|\lambda_{k}^{+}\right| \\
\zeta_{k}=-\operatorname{Re}\left(\log \lambda_{k}^{+}\right) f / \Omega_{k}
\end{gathered}
$$

The associated modal shape is equivalent to a column of the $C$ matrix of a complex diagonal realization of the system (see (19)).

The modal parameters of a mechanical system can thus be determined from a discrete time state-space model of the acceleration. If the mechanical structure is excited with an impulse or is released from an initial state, a state-space model can be identified from accelerometer measurements at selected points of the mechanical structure. 


\section{Identification Method}

Given measured data $y_{t}, u_{t}, t=0, \ldots, N-1$ from an experiment performed on the system, the aim of the identification process is to yield estimates of $A, B, C$ in (2) such that the fit to measured data

$$
V=\frac{1}{N} \sum_{t=0}^{N-1}\left|y_{t}-y(t)\right|^{2}
$$

is minimized. The minimization of $V$ in (6) is equal to minimize the prediction error criterion for an output-error model, see [15]. The focus will be on the case when the input is equal to an impulse signal

$$
u_{t}= \begin{cases}1, & t=0 \\ 0, & t>0\end{cases}
$$

and hence, the measured output is equal to the impulse response of the system.

The problem of estimating a linear model from impulse response data is equivalent to that of estimating the parameters of damped or undamped sinusoids hidden in noise, i.e., to fit the weighted sum (1) to a given set of noisy data $y_{t}$. The first solution to the problem is due to Prony [5] and variations of his method are described in several books on numerical analysis, $[13,7,10]$. More recent methods are the realization based algorithms, [23, $12,18,4]$, and optimal maximum likelihood methods, for undamped signals, [20]. Vibrational analysis applications using realization type algorithms are described in $[21,9,14]$. All these methods fits linear models to the data but do not minimize the prediction errors (6).

In this paper we will describe an approach to solve the stated minimization problem (6) by combining the subspace based realization algorithm by Kung [12] with standard prediction error minimization.

By merging these two very different approaches we obtain a method which utilize the advantages of both. The key idea is to use the non-iterative realization algorithms to obtain an initial estimate of a state-space model. If noise is present this model will be an approximation of the true system. The state-space model so obtained is then converted to some state-space realization appropriate for parameterization. Finally, the parameterized model is used as an initial estimate for the iterative prediction error method. Experience suggests that this last step can significantly improve the fit of the model to measured data. If the model order is high, the initial estimate provided by the realization algorithm step is crucial since the prediction error method, which involves a non-linear optimization, typically is trapped in local minima if the initial estimate is far from being able to describe the true system. 


\subsection{Identification with a Realization Algorithm}

The (block) Hankel matrix constructed from the impulse response of a finitedimensional linear system has some algebraic properties which can be used to construct a state-space realization. The basic property is that the rank of this Hankel matrix is equal to the order of the linear system which was noted in [11]. Full realization algorithms are described in [8, 22] and [19]. These algorithms is not applicable to real identification problems since all algorithms require noise-free data. In the noisy case the Hankel matrix is generically a full rank matrix.

In [23] a singular value decomposition of the Hankel matrix was introduced to approximate the full rank matrix with a low rank one and then use the techniques of the original realization algorithms. A similar approach also using the singular value decomposition is described in [12]. The eigensystem realization algorithm (ERA) by [9] is a slightly generalized version of the algorithm by Zeiger and McEwen [23]. Modal analysis by realization type algorithms has successfully been used for systems excited by non-stationary stochastic processes [3, 17]. The identification algorithm by Kung [12] has been used in this work and will briefly be reiterated in the following.

Assume we are given $N=i+j-1$ measurements $y_{t}$ of an impulse response. Construct the block-Hankel matrix

$$
H^{i j}=\left[\begin{array}{cccc}
y_{1} & y_{2} & \cdots & y_{j} \\
y_{2} & \vdots & \cdots & y_{j+1} \\
\vdots & \vdots & \vdots & \vdots \\
y_{i} & y_{i+1} & \cdots & y_{i+j-1}
\end{array}\right]
$$

where $i>n$ and $j \geq n$ is the number of block rows and columns respectively. Form the singular value decomposition

$$
H^{i j}=\left[\begin{array}{ll}
U_{1} & U_{2}
\end{array}\right]\left[\begin{array}{cc}
\Sigma_{1} & 0 \\
0 & \Sigma_{2}
\end{array}\right]\left[\begin{array}{l}
V_{1}^{T} \\
V_{2}^{T}
\end{array}\right]
$$

where the diagonal matrix $\Sigma_{1}$ contains the $n$ principal singular values. If we assume that $y_{t}$ is noise-free and originates from (2), $H^{i j}$ will be of rank $n$ and hence $\Sigma_{2}=0$. A realization of $(2)$ is then calculated as follows. Denote by $\underline{U}_{1}$ and $\bar{U}_{1}$ the sub-matrices formed from $U_{1}$ by removing the $p$ first and last rows respectively. The $\hat{A}$ matrix is given by

$$
\hat{A}=\left(\bar{U}_{1} \Sigma_{1}^{1 / 2}\right)^{\dagger} \underline{U}_{1} \Sigma_{1}^{1 / 2}
$$

where $(\cdot)^{\dagger}$ is the Moore-Penrose pseudo inverse. By introducing the selection matrices

$$
E_{p}^{T}=\left[\begin{array}{llll}
I_{p} & 0_{p} & \ldots & 0_{p}
\end{array}\right], \quad E_{m}^{T}=\left[\begin{array}{llll}
I_{m} & 0_{p} & \ldots & 0_{p}
\end{array}\right]
$$


where $I_{i}$ denotes the $i \times i$ identity matrix and $0_{i}$ denotes the $i \times i$ zero matrix, $\hat{B}$ and $\hat{C}$ are given by

$$
\hat{B}=\hat{\Sigma}_{1}^{1 / 2} \hat{V}_{1}^{T} E_{m}, \quad \hat{C}=E_{p}^{T} \hat{U}_{1} \hat{\Sigma}_{1}^{1 / 2}
$$

The estimated system $(\hat{A}, \hat{B}, \hat{C})$ is related to the original system $(A, B, C)$ by a similarity transformation and hence, their input-output properties coincide. If we use this algorithm on noisy data or data from a high order system (true order $>n) \hat{\Sigma}_{2}$ will not be identically zero and the system $(\hat{A}, \hat{B}, \hat{C})$ is an approximation of the true system. This approximation does not necessarily minimize (6). However, our practical experience is that models which are close to the minimum are provided by the algorithm.

\subsection{Prediction Error Minimization}

The prediction error identification methods (PEM) contain the most known and used methods for system identification and has been developed and analyzed extensively during the last three decades. For a unifying treatment we refer to [15]. Here we will concentrate on the state-space model (2). Since a noise model is not included, the model (2) is commonly referred to as an output-error form.

The state-space system matrices $(A, B, C)$ are parameterized using a parameter vector $\theta$ to obtain the parameterized predictor model

$$
\begin{aligned}
\hat{x}(t+1) & =A(\theta) \hat{x}(t)+B(\theta) u(t), \\
\hat{y}(t \mid \theta) & =C(\theta) \hat{x}(t)
\end{aligned}
$$

One possible parameterization, using a minimal number of parameters needed, is given by the multivariable identifiable forms, also called canonical forms, see [15]. The parameter vector $\theta$ and hence the model is obtained by minimizing the squared sum of the prediction errors

$$
\hat{\theta}=\arg \min _{\theta} \frac{1}{N} \sum_{t=1}^{N}\left|y_{t}-\hat{y}(t \mid \theta)\right|^{2}
$$

This minimization is in general nonlinear in the parameters $\theta$ and an analytical solution is not feasible. Hence, (14) has to be solved by an iterative

minimization method, e.g. a Gauss-Newton algorithm [6]. The success of this approach is to a large extent dependent on the initial estimate from which the iterative method is started. This fact becomes increasingly significant as model complexity grows.

\subsubsection{Linear Least-Squares Estimation}

From the initial estimate obtained from the realization algorithms it is fairly simple to improve the model quality by re-estimating the $C$ matrix from the 
estimation data by assuming $A$ and $B$ fixed. This can be done by means of minimizing the squared prediction error $y_{t}-\hat{y}(t)$. For fixed $\hat{A}$ and $\hat{B}$, the impulse response $\hat{y}(t)$ is a linear function of $C$. Thus, the estimation is easily accomplished by an ordinary least-squares solution.

Assume that $y_{t}, t=1, \ldots, N$, is the noisy impulse response. We can then write

$$
\left[\begin{array}{llll}
y_{1} & y_{2} & \cdots & y_{N}
\end{array}\right]=\hat{C} \hat{\mathcal{C}}_{N}+\left[\begin{array}{llll}
v_{1} & v_{2} & \cdots & v_{N}
\end{array}\right]
$$

where $\hat{\mathcal{C}}_{N}$ is the $N$ block row extended controllability matrix constructed from $\hat{A}$ and $\hat{B}$

$$
\hat{\mathcal{C}}_{N}=\left[\begin{array}{llll}
\hat{B} & \hat{A} \hat{B} & \cdots & \hat{A}^{N-1} \hat{B}
\end{array}\right]
$$

and $v_{i}$ the residuals. The least-squares solutions to estimate $C$ is

$$
\hat{C}=\left[\begin{array}{llll}
y_{1} & y_{2} & \ldots & y_{N}
\end{array}\right] \hat{\mathcal{C}}_{N}^{\dagger}
$$

where $(\cdot)^{\dagger}$ is the Moore-Penrose pseudo-inverse. If $(\hat{A}, \hat{B})$ is a controllable pair $\hat{\mathcal{C}}_{N}$ will be of full rank $n$ and the solution $\hat{C}$ unique.

A completely dual approach is obtained if we consider the estimation of $B$, assuming $\hat{A}$ and $\hat{C}$ fixed.

Either of these least-square steps will move the model closer to the prediction error minimum (6) and thus improve the speed of convergence for the final step, the non-linear iterative prediction error minimization.

\subsubsection{Numerically Effective Identifiable Parameterizations}

The state-space model, obtained from the realization algorithm and further refined with the least-squares estimation, is now an excellent initial model for prediction error minimization. In order to perform the non-linear optimization by the Gauss-Newton method we need a parameterization of the state-space model. The parameterization should be designed with the focus on vibrational analysis applications. The characteristics in the case here under study are single-input system with a large number of complex poles as well as a large number of outputs.

During the prediction error minimization using a Gauss-Newton method the main computational effort lies in calculating the gradient and in the search for a lower value of the criterion along the Gauss-Newton direction. From (13) notice that the gradient of $\hat{y}(t \mid \theta)$ with respect to the elements in the $C$ matrix is equivalent to the state $\hat{x}(t)$. Henceforth, this gradient calculation is easily accomplished. For our case with many outputs an advantageous parameterization uses many parameters in the $C$ matrix.

Since we are dealing with self adjoint systems the $A$ matrix can be made diagonal by a change of state-space basis and we can derive a parameterization which is both minimal, numerically efficient and well conditioned. 


\subsubsection{An Identifiable Parameterization}

Consider a minimal system $(A, B, C)$ with a non-deficient $A$ matrix and $m=$

1 (single input system). Then there exists a non-singular matrix $T \in \mathbb{C}^{n \times n}$ such that

$$
T^{-1} A T=\Lambda=\operatorname{diag}\left(\lambda_{1}, \lambda_{2}, \ldots, \lambda_{n}\right),
$$

where $\lambda_{i} \in \mathbb{C}$ are the poles of the system. We also impose a total ordering of the eigenvalues such that the primary ordering is by the imaginary part and the secondary ordering is based on the real part. Since $A$ is a real matrix, for each complex eigenvalue $\lambda_{i}$ there exists a $j \neq i$ such that $\lambda_{i}=\lambda_{j}^{*}$ where $(\cdot)^{*}$ denotes complex conjugate. If we use $T$ as the complex similarity transformation, we obtain the system

$$
\left(\Lambda, B_{c}, C_{c}\right)=\left(T^{-1} A T, T^{-1} B, C T\right)
$$

in the complex diagonal state-space form. The complex diagonal form (19) does not provide an identifiable parameterization since the diagonal form is preserved by diagonal non-singular similarity transformations

$$
T=\operatorname{diag}\left(t_{1}, \ldots, t_{n}\right) .
$$

and is thus non-unique. An identifiable parameterization is obtained if we add the constraint

$$
B_{c}=[1, \ldots, 1]^{T}
$$

on the system. The minimality of the system implies that this choice of $B_{c}$ always is possible. The two conditions, the ordered diagonal form of $\Lambda$ and (21), make this form identifiable since it is not possible to find any similarity transformation which preserves this structure.

If we consider a general multivariable system with non-repeated poles, the described diagonal form can easily be extended to multi-input systems under the assumption that the system is controllable from the first input. An identifiable parameterization is then obtained by introducing parameters for all rows and from the second up to the $m$ th column of $B_{c}$, while keeping fixed ones in the first column. In this case, the total number of parameters becomes $n(p+m)$. The controllability requirement from the first input can be relaxed if a more complex method of fixing ones in the $B_{c}$ matrix is devised. This leads to a set of non-overlapping parameterizations.

The main advantages of this type of parameterization is the simple coupling between parameters and poles of the system as well as, for a free decaying system, relative amplitude and phases between different outputs. The decoupling of the states also, as stated before, leads to a low computational load. Disadvantages are the restriction to systems which have nonrepeated poles and, for the multi-input case, that the system is required to be controllable from the first input. 


\subsubsection{Block Diagonal Identifiable Parameterization}

It is sometimes required, (usually for implementation reasons), that the parameterization results in a state-space system with real-valued matrices. It is straight forward to derive such a form from the complex diagonal realization (19) with the added constraint on $B_{c}(21)$. The columns of $C_{c}$ corresponding to real-valued eigenvalues are also real-valued and thus need no change.

For the sub-system corresponding to complex eigenvalues we can obtain a block diagonal form by employing a block diagonal similarity transformation matrix

$$
T_{b}=\frac{1}{\sqrt{2}} \operatorname{diag}\left(\left[\begin{array}{cc}
1 & i \\
1 & -i
\end{array}\right], \ldots,\left[\begin{array}{cc}
1 & i \\
1 & -i
\end{array}\right]\right) .
$$

With the transformation above the block diagonal sub-system results in a realization

$$
\left(A_{b}, B_{b}, C_{b}\right)=\left(T_{b}^{-1} \Lambda T_{b}, T_{b}^{-1} B_{c}, C_{c} T_{b}\right) .
$$

Here $A_{b}$ is a block diagonal matrix where each block element has the form

$$
\left\{A_{b}\right\}_{k k}=\left[\begin{array}{cc}
\operatorname{Re}\left(\lambda_{k}^{+}\right) & -\operatorname{Im}\left(\lambda_{k}^{+}\right) \\
\operatorname{Im}\left(\lambda_{k}^{+}\right) & \operatorname{Re}\left(\lambda_{k}^{+}\right)
\end{array}\right]
$$

where $\left\{\lambda_{k}^{+}\right\}$are the $n / 2$ eigenvalues with positive imaginary parts. The $B_{b}$ matrix is of the form

$$
B_{b}=\left[\begin{array}{lllllll}
1 & 0 & 1 & 0 & \ldots & 1 & 0
\end{array}\right]^{T} .
$$

The $C_{b}$ matrix becomes real but without any particular structure. The number of parameters in this structure is $n(p+1)$ as before.

Example 3.1 A fourth order system with two outputs has a block diagonal parameterization given by

$$
\begin{aligned}
x(t+1) & =\left[\begin{array}{cccc}
\theta_{1} & -\theta_{2} & 0 & 0 \\
\theta_{2} & \theta_{1} & 0 & 0 \\
0 & 0 & \theta_{3} & -\theta_{4} \\
0 & 0 & \theta_{4} & \theta_{3}
\end{array}\right] x(t)+\left[\begin{array}{l}
1 \\
0 \\
1 \\
0
\end{array}\right] u(t) \\
y(t) & =\left[\begin{array}{cccc}
\theta_{5} & \theta_{7} & \theta_{9} & \theta_{11} \\
\theta_{6} & \theta_{8} & \theta_{10} & \theta_{12}
\end{array}\right] x(t)
\end{aligned}
$$

\subsection{System Order Selection}

An important user choice is the number of states, or modes, to use in (2). A possible guideline is to choose the model order equal to the number of 
"large" singular values of the Hankel matrix ( 8 since the rank is equal to the model order in the noise free case. However, the presence of noise and structural nonlinearities usually make the Hankel matrix (8) of full rank. However, if a clear gap exists among the largest and the smallest singular values of $(9)$, it is natural to regard the largest singular values as originating from the linear system and the smallest ones from noise and nonlinearities. Other evaluation criteria usually plays an even more important part in the the final choice of model order and this will be the topic of the next section.

\section{Evaluation Criteria}

In the process of deriving accurate models, validation plays an important role. In the validation step estimated models are evaluated in the light of other information than used in the estimation step. Typically a second independent data set is used to determine the quality of the estimated model. If the result is not satisfying new models, often of different order, is reestimated and again evaluated until satisfactory models have been found.

In this section we will describe two different methods suited for model quality evaluation. First a cross-validation technique will be described which instead of using an independent data set (in time) will use an independent output channel set (in space). Secondly, we introduce the Modal Coherence Indicators. These indicators, one for each estimated pole or complex pole pair, indicate the quality of each mode by comparing the measured impulse response to the estimated model.

\subsection{Cross-Validation}

If several experiments are performed on the same system under similar conditions one set of measurements can be used for estimation and the other independent set to validate the model. When only one data set is available but consists of a large number of output channels (here accelerometer measurements) we can divide the outputs into two disjoint sets. If the division is made in such a way that all modes are present in both sets we can estimate a model using only one set of output channels and validate using the other set. In this paper we have used this latter method.

Assume the estimated model is given by $(\hat{A}, \hat{B}, \hat{C})$ derived using the es-

timation output set. To validate this model, we estimate $\hat{C}_{v}$ using the validation outputs. Since the matrix is linear in the outputs, given $\hat{A}$ and $\hat{B}$, $\hat{C}_{v}$ is calculated by minimizing (6) using an ordinary least-squares solution.

As a measure of model quality we will use the sample prediction error variance

$$
\hat{\bar{V}}=\frac{1}{N} \sum_{t=1}^{N}\left|y_{t}-\hat{y}(t \mid \hat{\theta})\right|^{2}
$$


which equals the residuals from the estimation of $\hat{C}_{v}$. Since $C_{v}$ is estimated using the validation channels, only the frequencies and damping ratios can be considered validated using this method.

\subsection{Modal Coherence Indicators}

The most straight-forward way to determine the validity of a state-space model $(A, B, C)$ with respect to a measured empirical impulse response is simply to calculate the impulse response of the state-space model and compare it with the empirical one, e.g. calculate the norm of the errors. This type of measure only describes the total performance of the model and gives no information about which modes of the model are accurate and which are not. The modes are easily accessible from the eigenvalues of the $A$ matrix but they are fairly hidden in the empirical impulse response unless a model is estimated from the impulse response. Hence, it is somewhat difficult to obtain a distributed measure which describes the quality of each of the modes of the system $(A, B, C)$ by comparing the empirical impulse response and the system $(A, B, C)$ to each other without explicitly estimating a model.

The Modal Amplitude Coherence, introduced by Juang et al. [9] is one approach to obtain quality measures connected to each of the estimated modes of the system. This measure uses the controllability matrix of the estimated state-space model and is only defined for state-space models derived from the realization algorithm by Kung [12] or Zeiger-McEwen (ERA) [23]. Here we will generalize this measure for arbitrary estimation methods and present a practical method of how to calculate it.

Assume an impulse response $\{y(t)\}_{t=1}^{N}$ and a state-space realization $(A, B, C)$ is given. For one model a set of Modal Coherence Indicators $\gamma_{k}, k=1, \ldots, n$ is determined. Each indicator $\gamma_{k}$ is associated with one eigenvalue $\lambda_{k}$ of the matrix $A$. The set of indicators $\gamma_{k}$ is calculated by comparing the extended observability and controllability matrices from the model $(A, B, C)$ with the extended observability and controllability matrices directly derived from the empirical impulse response using the complex diagonal form as a state-space basis.

A fundamental problem in the comparison is that the controllability and observability matrices must be converted to a unique common basis in order to make any meaningful comparison. In what follows we will introduce a state-space realization in a normal form as well as a normal form of a singular value decomposition of a matrix. These normal forms form a common unique description basis which can be transformed to the complex diagonal form.

Let the extended observability and controllability matrices $\mathcal{O}_{i}$ and $\mathcal{C}_{j}$ be 
defined as

$$
\begin{gathered}
\mathcal{O}_{i}=\left[\begin{array}{c}
C \\
C A \\
\vdots \\
C A^{i-1}
\end{array}\right] \\
\mathcal{C}_{j}=\left[\begin{array}{llll}
B & A B & \cdots & A^{j-1} B
\end{array}\right]
\end{gathered}
$$

with the dimensions $\mathcal{O}_{i} \in \mathbb{R}^{p i \times n}$ and $\mathcal{C}_{j} \in \mathbb{R}^{n \times m j}$.

Definition 4.1 A state-space model $(A, B, C)$ is in $i j$-balanced normal form if the $i$-extended observability matrix (28) and $j$-extended controllability matrix (29) satisfy the relations

$$
\mathcal{O}_{i}^{T} \mathcal{O}_{i}=\mathcal{C}_{j} \mathcal{C}_{j}^{T}=\Sigma
$$

where $\Sigma=\operatorname{diag}\left(\sigma_{1}, \sigma_{2}, \ldots, \sigma_{n}\right)$ with $\sigma_{1} \geq \sigma_{2} \geq \ldots \geq \sigma_{n} \geq 0$ and if the first non-zero element in each column of $\mathcal{O}_{j}$ is positive.

A minimal system can always be transformed to the $i j$-balanced normal form, see [16].

Definition 4.2 The SVD factorization (9) is a normal form singular value decomposition if $\Sigma_{1}=\operatorname{diag}\left(\sigma_{1}, \sigma_{2}, \ldots, \sigma_{n}\right)$ with $\sigma_{1}>\sigma_{2}>\ldots>$ $\sigma_{n}>0$ and the first non-zero element in each column of $U_{1}$ is positive.

Consider the SVD (9) of the Hankel matrix $H^{i j}$ constructed from the given impulse response satisfying the sign condition in Definition 4.2. Then

$$
\mathcal{O}_{i}^{d}=U_{1} \Sigma_{1}^{1 / 2}
$$

and

$$
\mathcal{C}_{j}^{d}=\Sigma_{1}^{1 / 2} V_{1}^{T}
$$

are the observability and controllability matrices of a system of order $n$ directly inferred from the given impulse response. Notice that

$$
\mathcal{O}_{i}^{d^{T}} \mathcal{O}_{i}^{d}=\mathcal{C}_{j}^{d} \mathcal{C}_{j}^{d^{T}}=\Sigma_{1}
$$

which we, from Definition 4.1, recall as $i j$-balanced normal form. Hence we can say that the data-inferred observability and controllability matrices are given in $i j$-balanced normal form. The two normal forms constitute a common description basis with the following property:

Lemma 4.1 Consider an ij-balanced normal form $(A, B, C)$ with the extended observability and controllability matrices $\mathcal{O}_{i}$ and $\mathcal{C}_{j}$, respectively, and 
a normal form SVD factorization as in Definition 4.2 of a Hankel matrix $H^{i j}$ with non-repeated singular values $\Sigma_{1}$. Then

$$
\mathcal{O}_{j}=U_{1} \Sigma_{1}^{1 / 2} \text { and } \mathcal{C}_{j}=\Sigma_{1}^{1 / 2} V_{1}^{T}
$$

if and only if $H^{i j}$ is constructed from the impulse response of the system $(A, B, C)$.

Proof. See [16]

The result above indicates a way of comparing implicit models given in impulse response form with explicit models in state-space form by comparing the observability and controllability matrices respectively.

Consider again the complex diagonal form $\left(\Lambda, B_{c}, C_{c}\right)$ from (19). This realization is particularly interesting since the modes of the systems can be associated with the diagonal elements of $\Lambda$, which are the poles of the system. Furthermore, row $k$ of the $B_{c}$ matrix and column $k$ of the $C_{c}$ matrix are associated with the $k$ th pole of the system. We can thus write the transfer function as the sum

$$
G(z)=\sum_{k=1}^{n} \frac{1}{z-\lambda_{k}} C_{c}^{k} B_{c}^{k},
$$

where the superscript denotes column and row numbers, respectively.

Recall that the estimated system $(A, B, C)$ given in $i j$-balanced normal form has the observability and controllability matrices $\mathcal{O}_{i}$ and $\mathcal{C}_{j}$, respectively. If $T$ is the complex similarity transformation which diagonalizes $A$, the corresponding complex observability and controllability matrices are

$$
\mathcal{O}_{i}^{c}=\mathcal{O}_{i} T, \quad \mathcal{C}_{j}^{c}=T^{-1} \mathcal{C}_{j} .
$$

Note that the $k$ th column of $\mathcal{O}_{i}^{c}$, as well as the $k$ th row of $\mathcal{C}_{j}^{c}$, is associated with the $k$ th eigenvalue $\lambda_{k}$.

Consider the normal form SVD factorization (9) of the Hankel matrix $H^{i j}$. If the Hankel matrix is constructed from the impulse response of the system $(A, B, C)$, it is clear from Lemma 4.1 that

$$
\mathcal{O}_{i}^{c}=U_{1} \Sigma_{1}^{1 / 2} T \text { and } \mathcal{C}_{j}^{c}=T^{-1} \Sigma_{1}^{1 / 2} V_{1}^{T},
$$

where $T$ is given by (18).

From the two relations (37) we can for each eigenvalue $\lambda_{k}$ compare the columns of the observability relation with each other and the rows of the controllability relation.

Definition 4.3 The modal observability coherence $\gamma_{k}^{o}$ is defined as

$$
\gamma_{k}^{o}=\frac{\left|\left(\mathcal{O}_{i}^{c} e_{k}\right)^{H} U_{1} \Sigma_{1}^{1 / 2} T e_{k}\right|}{\left(\left|\left(\mathcal{O}_{i}^{c} e_{k}\right)^{H} \mathcal{O}_{i}^{c} e_{k}\right|\left|\left(U_{1} \Sigma_{1}^{1 / 2} T e_{k}\right)^{H} U_{1} \Sigma_{1}^{1 / 2} T e_{k}\right|\right)^{1 / 2}}, k=[1, \ldots, n] .
$$


The modal controllability coherence $\gamma_{k}^{c}$ is defined as

$$
\gamma_{k}^{c}=\frac{\left|e_{k}^{T} \mathcal{C}_{j}^{c}\left(e_{k}^{T} T^{-1} \Sigma_{1}^{1 / 2} V_{1}^{T}\right)^{H}\right|}{\left.\left|e_{k}^{T} \mathcal{C}_{j}^{c}\left(e_{k}^{T} \mathcal{C}_{j}^{c}\right)^{H}\right|\left|e_{k}^{T} T^{-1} \Sigma_{1}^{1 / 2} V_{1}^{T}\left(e_{k}^{T} T^{-1} \Sigma_{1}^{1 / 2} V_{1}^{T}\right)^{H}\right|\right)^{1 / 2}}, k=[1, \ldots, n]
$$

where $\mathcal{O}_{i}^{c}$ and $\mathcal{C}_{j}^{c}$ are given by $(36)$ and $U_{1}, \Sigma_{1}$ and $V_{1}$ are given by (9) and $\mathrm{T}$ by (18) and $e_{k}$ is a unit vector with a one in position $k$.

Furthermore, the Modal Coherence Indicator is defined as

$$
\gamma_{k}=\gamma_{k}^{c} \gamma_{k}^{o}, \quad k=1, \ldots, n
$$

Given a state-space model and an impulse response the set $\left\{\gamma_{k}\right\}_{k=1}^{n}$ of Modal Coherence Indicators describes the "coherence" between the modes of the state-space model and the impulse response and thus serves as a distributed model quality measure.

We can now state the following theorem.

Theorem 4.1 The properties of the Modal Coherence Indicators from Definition 4.3 are

$$
\gamma_{k} \in[0,1], \forall k \in[1,2, \ldots, n]
$$

and if the Hankel matrix $H^{i j}$ and the system $(A, B, C)$ share the same impulse response coefficients we have

$$
\gamma_{k}=1, \forall k \in[1,2, \ldots, n] .
$$

Proof. The result follows trivially from Definition 4.3 and Lemma 4.1.

The modal controllability coherence measure from Definition 4.3 is equal to the Modal Amplitude Coherence introduced in [9] if the state-space model $(A, B, C)$ originates from the algorithm by Zeiger-McEwen or the ERAalgorithm. The novelty of the introduced method is that given any statespace model and an impulse response, the Modal Coherence Indicators can be calculated.

Depending on how the state-space model is estimated the observability and controllability modal coherence can yield quite different results in the case of noisy impulse response data. The best choice is then to use the Modal Coherence Indicators as defined by (40). A high Modal Coherence Indicator is then equal to a high observability as well as a high controllability coherence.

From the definition it follows that $n$ Modal Coherence Indicators (MCIs) can be calculated from a system of order $n$. For a complex conjugate eigenvalue pair the two corresponding MCIs will be equal. For one vibrational mode we thus only need to consider one MCI. For real eigenvalues each eigenvalue has a unique MCI. 


\subsubsection{Stabilization Diagram}

The Modal Coherence Indicators (40) can be used as a quality tag attached to each of the vibrational modes of an estimated model. Each vibrational mode is characterized, among other things, by the frequency $\omega_{k}(3)$. The indicators $\gamma_{k}$ can be used in a diagram to visualize frequency location and modal accuracy. Such a diagram is called a stabilization diagram. In a stabilization diagram the abscissa axis represents frequency and the ordinate Modal Coherence. A vertical bar of length $\gamma_{k}$ is plotted for each mode at the frequency location given by $\omega_{k}$. If several models of different orders are available the corresponding bar graphs can be stacked on top of each other, starting with the model with the lowest order.

The characteristic of the diagram is that for identified models with too low order some frequencies (modes) give rise to low Modal Coherence Indicators (the bars are short). As the model order increases these split into two or more modes with higher indicators (or disappear again). As the model order increases the identified frequencies "stabilize", hence the name stabilization diagram.

\section{The Experiments}

The experimental results presented here are part of a large-scale experimental damping survey performed on the Saab 2000 aircraft. The study was aimed at revealing the damping properties and their dependence on deformation of a body-in-green fuselage/wing/nacelle assembly (see Figure 1). The nacelle is the outer casing of the engine. It was suspected before the test, and verified and quantified by the test, that the damping would increase with increasing vibrational magnitude. The test was divided into two phases, the first consisting of a conventional ground vibration (normal mode) test at a low vibrational level and under stationary harmonic conditions. The second phase was a complementary high vibrational level study. The results of the test were to be applied in the aircraft load evaluation and simulation of extremely hard landings (up to $3 \mathrm{~m} / \mathrm{s}$ sink rate). The results presented in this chapter are only from the second phase of the test.

Various excitation locations and magnitudes were used during the second phase. Snap-back excitations from pre-determined deflection states were used as structural inputs. Accelerometers and load cells were used to sense the structural response. An enormous amount of data were collected during the test, out of which a selected amount has been used in this section. The results presented here were obtained during a wing-tip snap-back excitation from a moderate initial deflection state $(10 \%$ of the highest excitation level used). In this test 21 accelerometers were distributed over the wings (12 accelerometers), the nacelles (6) and fuselage (3). Load cells were used to

register the reactive loads on the supports and tension in the pre-stressed 


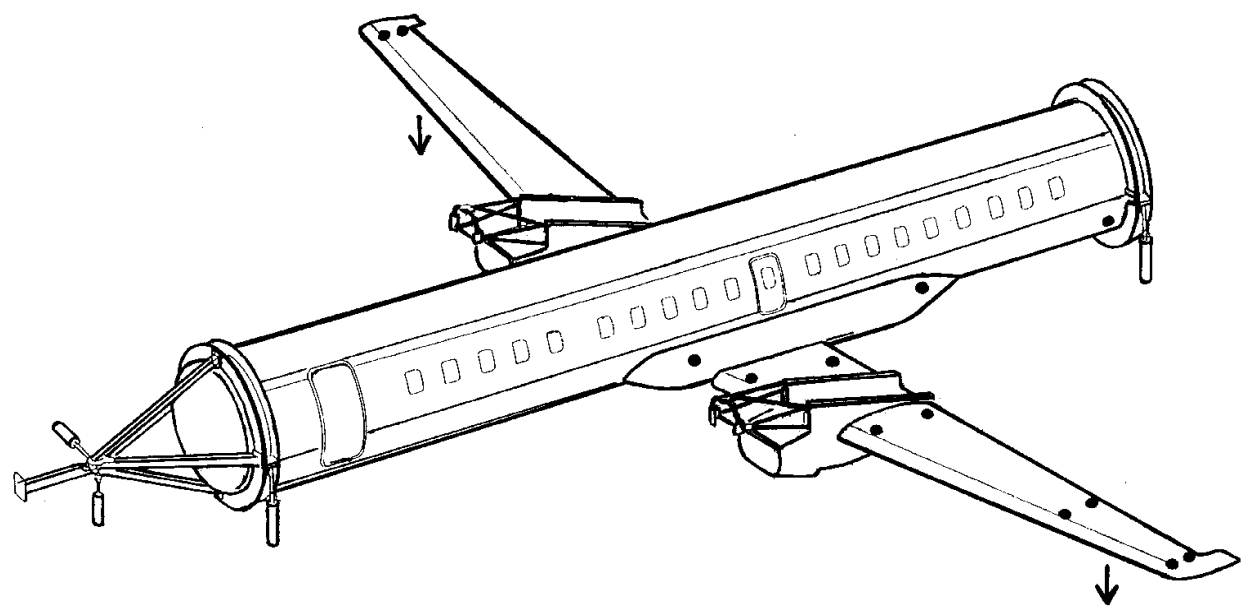

Figure 1: Test specimen. Location of wing and fuselage accelerometers are shown as dots (nacelle accelerometers are not shown). All shown accelerometers sense vertical accelerations. Arrows indicate location of the snap-back cables.

cables used for excitation. A sampling rate of $512 \mathrm{~Hz}$ was used and a total 16384 samples collected.

\subsection{Data pre-processing}

The acceleration response to the snap-back excitation is triggered by the almost instantaneous release of cable tension. Thereafter the accelerations are governed by the free-decay properties of the structure. In the identification procedure we considered the data as originating from an impulse excitation.

To reduce the amount of data, the measured acceleration signals were decimated with a factor of two to a sample rate of $256 \mathrm{~Hz}$. To illustrate the properties of the accelerometer signals a selection of measured channels is shown in Figure 2. In the right hand graphs the first 1200 samples are shown. The left graphs focus on the first 250 samples. We notice a large amount of resonant modes with frequencies distributed in a rather wide range.

Based on visual inspection, the measured signals to be used in the identification were chosen to be sample numbers 26-1250. By choosing sample 26 as the first sample we assure that the snap back excitation has fully taken place. The output channels were divided into the two disjoint sets of estimation data and validation data. The choice was based on the modal contents of each channel. The channels were grouped into 4 groups were each group represented low, low to medium, medium and high frequency content. This choice was also based on visual inspection of the 21 output channels. The validation and estimation channels were then selected by drawing from all 

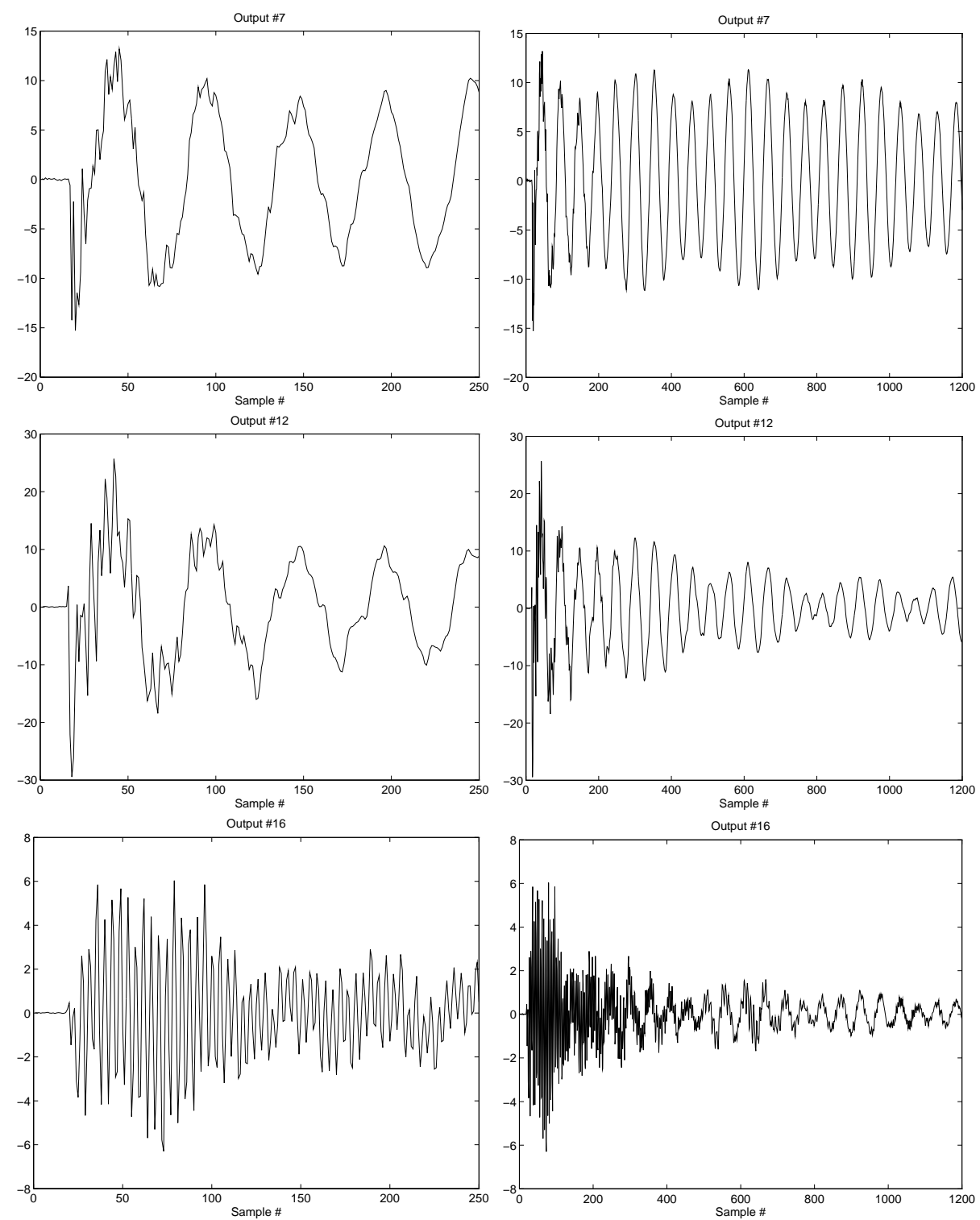

Figure 2: A selection of measured accelerations at different points of the test specimen from the snap-back excitation experiment. Notice the large amount of modes with frequencies distributed in a rather wide range. Left graphs show the first 250 samples of the corresponding graphs to the right. The data shown is after the decimation step and thus corresponds to a sampling frequency of $256 \mathrm{~Hz}$. 


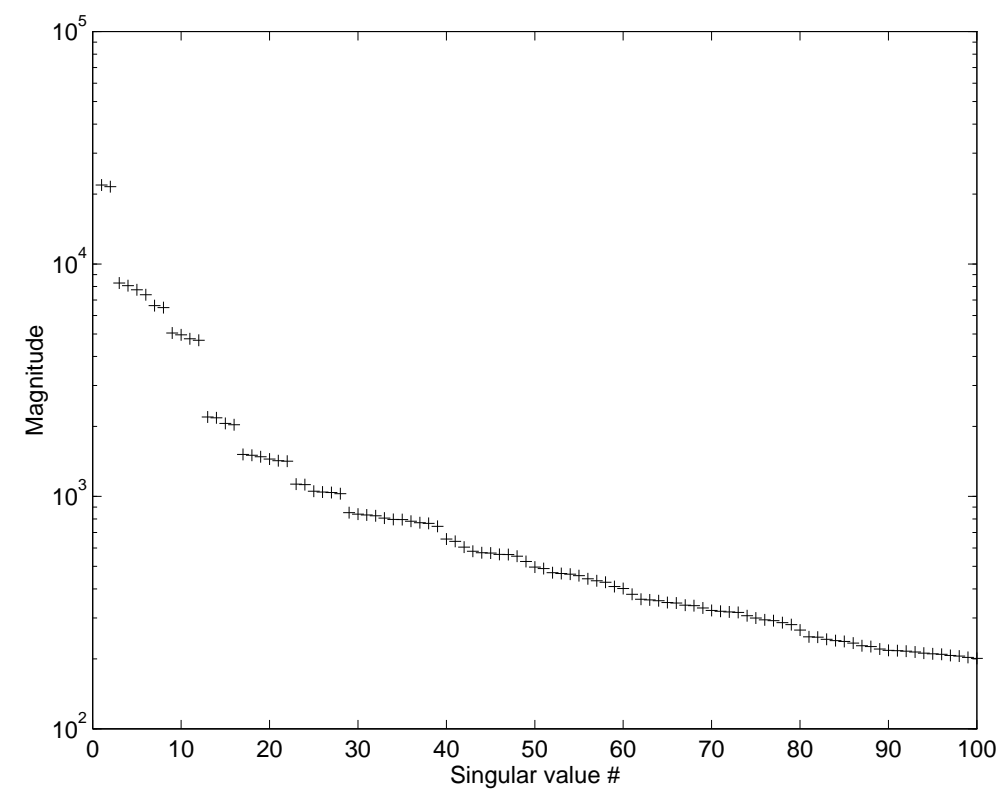

Figure 3: The graph shows the 100 largest singular values of the Hankel matrix based on the estimation data.

four groups. In this way we ensured approximately the same modal contents in both data sets.

The estimation data set contains 14 accelerometer channels each consisting of 1225 samples. The validation data set contains 7 channels also with 1225 samples each. The two data sets were normalized in such a way that all signals were of equal 2-norm.

\subsection{Identification of Vibrational State-Space models}

In a first step we apply Kung's algorithm, from Section 3.1. The major computational part consists of calculating the SVD of the Hankel matrix (8). To reduce noise influence we choose to select $i=80$ as the number of block rows of $H^{i j}$. This choice makes the Hankel matrix almost square. For validation purposes we also calculate the SVD of the Hankel matrix of the validation data with $i=80$. The magnitudes of the 100 largest singular values of the Hankel matrix based on the estimation data are depicted in Figure 3. From the figure we can draw the conclusion that a model order in the range from 10 to 30 should be considered.

In the next step, which is aimed at determining the appropriate model order we focus on both the Modal Coherence Indicators from Definition 4.3 and cross-validation techniques. For increasing model orders we estimate 

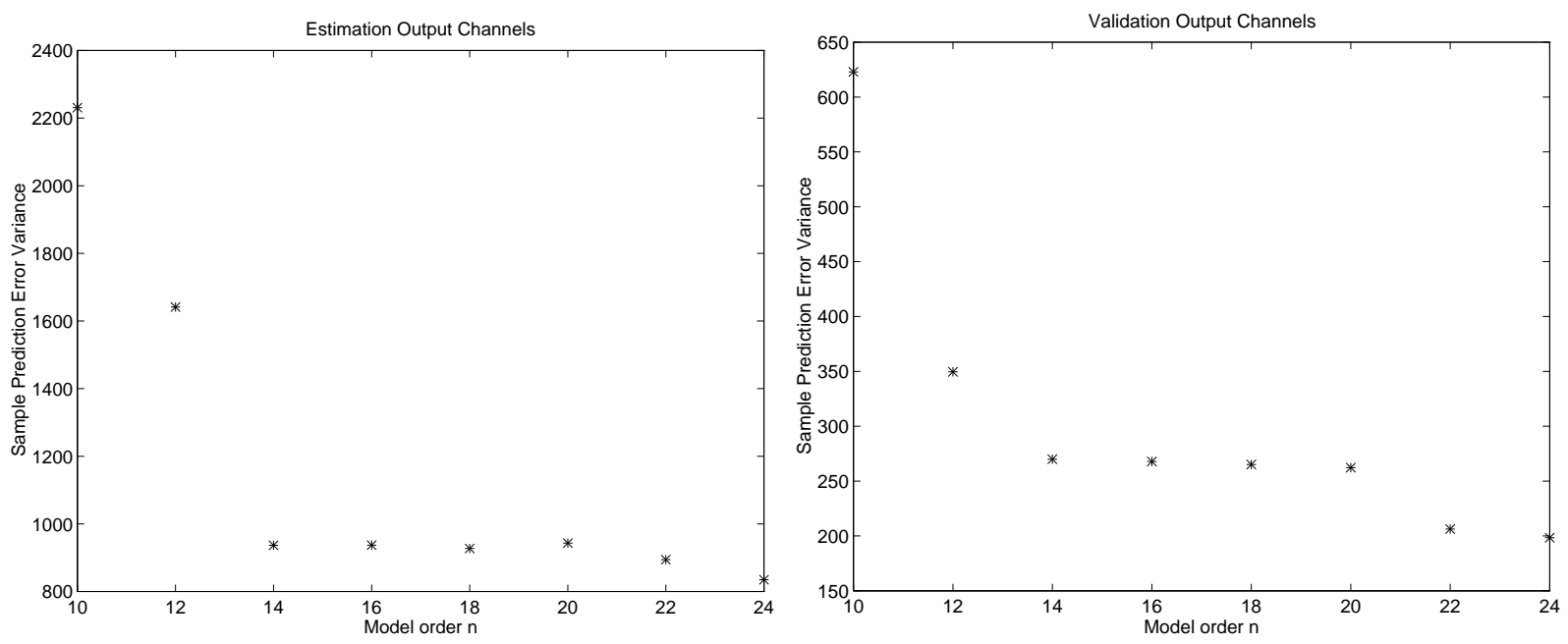

Figure 4: $\hat{\bar{V}}$ calculated from the estimation data set (left graph) and from the validation data set (right graph). In the graphs the result from estimated models of orders 10 to 24 are depicted. The models are estimated using Kung's algorithm followed by a re-estimation of the $C$ matrix.

models $(A, B, C)$ from the estimation data using Kung's algorithm and employ least-squares estimation of the $C$ matrix to refine the models. Recall that the SVD is already calculated so each new estimation is almost instantaneous. Each estimated model is validated by re-estimating a $C$ matrix, denoted by $C_{v}$, now using the validation data set. As a quality measure we use

$$
\hat{\bar{V}}=\frac{1}{N} \sum_{k=1}^{N}\left|g_{k}-\hat{g}_{k}\right|^{2}
$$

where $\left\{g_{k}\right\}$ is the validation data set and $\left\{\hat{g}_{k}\right\}$ is the impulse response from the state-space model $\left(A, B, C_{v}\right)$. Also for each estimated model we calculate the Modal Coherence Indicators (MCI) $\gamma_{k}$ using the estimated model $\left(A, B, C_{v}\right)$ and the validation data set. In Figure 4 the resulting $\hat{\bar{V}}$ for models of even order from 10 to 24 is shown. The left graph depicts $\hat{V}$ calculated on the estimation data while the right graph shows $\hat{\bar{V}}$ calculated on the validation data. From the two graphs it is not completely clear which model order seems to be appropriate. From both graphs we can however conclude that a model order of at least 14 is required. A possible candidate is also 22 since $\hat{V}$ calculated from the validation data is significantly reduced for this model order.

In Figure 5 two stabilization diagrams are shown. The left diagram is calculated using the estimation data and the right one using the validation data. Each diagram is composed of a sequence of bar graphs. For each 

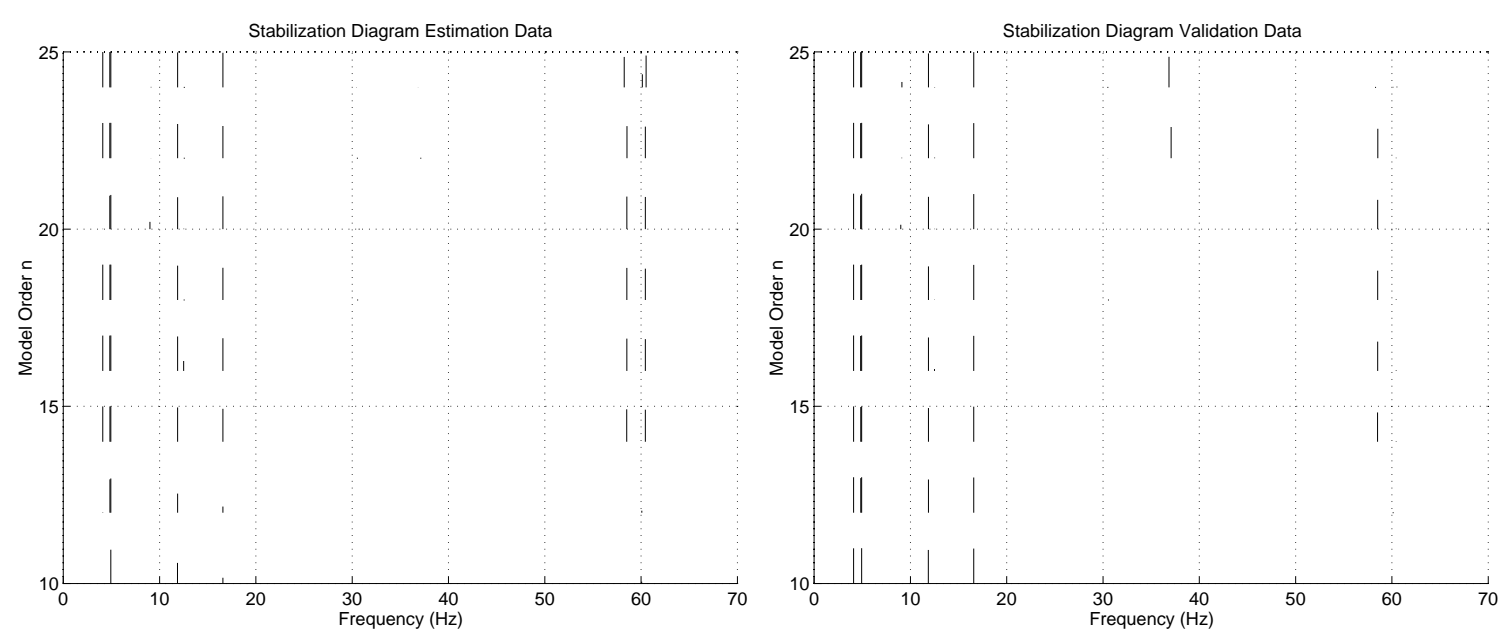

Figure 5: Stabilization diagram calculated from the estimation data and the estimated models (left graph) and from validation data (right graph). In the diagrams estimated models of order 10 to 24 are shown. The models are obtained by employing Kung's algorithm followed by least-squares estimation of the $C$ matrix. For each model order a bar graph is shown. The location of the bars along the abscissa is determined by the frequencies of the modes in the model. The length of each bar is equal to the corresponding MCI $\gamma_{k}$. In the figure the bar graphs for different model orders are placed on top of each other and the ordinate axis shows the corresponding model order.

estimated model a bar graph is drawn. The position of the bars along the abscissa is determined by the frequency of the estimated modes. The length of each bar is equal to $\gamma_{k}$, the corresponding MCI. A tall bar (maximum is 1) indicates modes of high quality. In the left diagram which corresponds to the estimation data we can identify 7 modes with high MCI for models of order 14 and higher. In the right graph corresponding to the validation data the same modes except one also have high indicators. A possible explanation to why the MCI for the mode at $60 \mathrm{~Hz}$ is low is that the validation channels do not contain this mode. Hence, the division between estimation and validation channels is not appropriate. For models with orders larger and equal to 22 a high quality mode appears in the right diagram. This might indicate that the validation channels contain a mode which is not very significant in the estimation data.

Based on this we decide to use model order 14 in the subsequent identification work since it adequately models the 7 dominant modes.

Once the model order is decided upon we now focus on prediction error minimization by parameterizing the model and perform a Gauss-Newton optimization. We use all 21 accelerometer channels to obtain the best possible model given the data at hand. A new Hankel matrix is constructed with 


\begin{tabular}{|r|rrr|}
\hline & Kung's alg. & $C$ re-est. & PEM \\
\hline$\hat{V}$ & 1235 & 1221 & 1177 \\
\hline
\end{tabular}

Table 1: Model quality for estimated models of order 14. The quality measure $\hat{\bar{V}}(43)$ is the average squared 2-norm of the difference between the measured signals and the model.

\begin{tabular}{|c|c|c|c|c|c|}
\hline $\begin{array}{r}\text { Kung's alg } \\
f(\mathrm{~Hz})\end{array}$ & $\zeta(\%)$ & MCI $\gamma$ & $\begin{array}{r}\text { PEM } \\
f(\mathrm{~Hz})\end{array}$ & $\zeta(\%)$ & MCI $\gamma$ \\
\hline 4.1031 & 0.4594 & 0.9980 & 4.1056 & 0.4685 & 0.9985 \\
\hline 4.8445 & -0.0402 & 0.9974 & 4.8376 & 0.0264 & 0.9966 \\
\hline 4.9398 & 0.4512 & 0.9994 & 4.9420 & 0.4139 & 0.9997 \\
\hline 11.8644 & 1.1942 & 0.9910 & 11.8756 & 1.1677 & 0.9861 \\
\hline 16.5732 & 1.1685 & 0.9891 & 16.5857 & 1.1580 & 0.9960 \\
\hline 58.4829 & 1.1304 & 0.9536 & 58.3674 & 1.3413 & 0.9560 \\
\hline 60.4157 & 0.3013 & 0.9730 & 60.4292 & 0.4157 & 0.9850 \\
\hline & Average: & 0.9859 & & Average: & 0.9883 \\
\hline
\end{tabular}

Table 2: Modal parameters of estimated model of order 14. All 21 output channels have been used in the estimation. Notice that one mode, $f=4.84$, of the the initial model delivered by Kung's algorithm is unstable.

the number of block rows $i=55$. Kung's algorithm is employed to estimate a model of order 14 and followed by the least-squares estimation of $C(17)$. This initial model is transformed to the block-diagonal form (23) to allow a minimal parameterization. This model structure has 14 parameters in the block diagonal $A$ matrix and $14 \times 21$ parameters in the $C$ matrix which yields a total of 308 parameters to be estimated. This might seem as a very large amount of parameters for a non-linear optimization. However, since the major part of the parameters originate from the $C$ matrix, the corresponding gradient calculations for these parameters are particularly simple. The results from the estimation are presented in the next section.

\subsection{Results}

The results from the three subsequent identification steps are reported in Table 1. Calculated on all data the loss function $\hat{\bar{V}}$ from (43) is reduced from the initial value of 1235 to 1177 by the least-squares estimation of $C$ followed by the prediction error minimization. In relative terms this constitutes a reduction by $4.7 \%$.

In Table 2 the estimated modal frequencies, damping ratios and corresponding MCIs are presented. Notice that one mode, $f=4.84$, of the the 

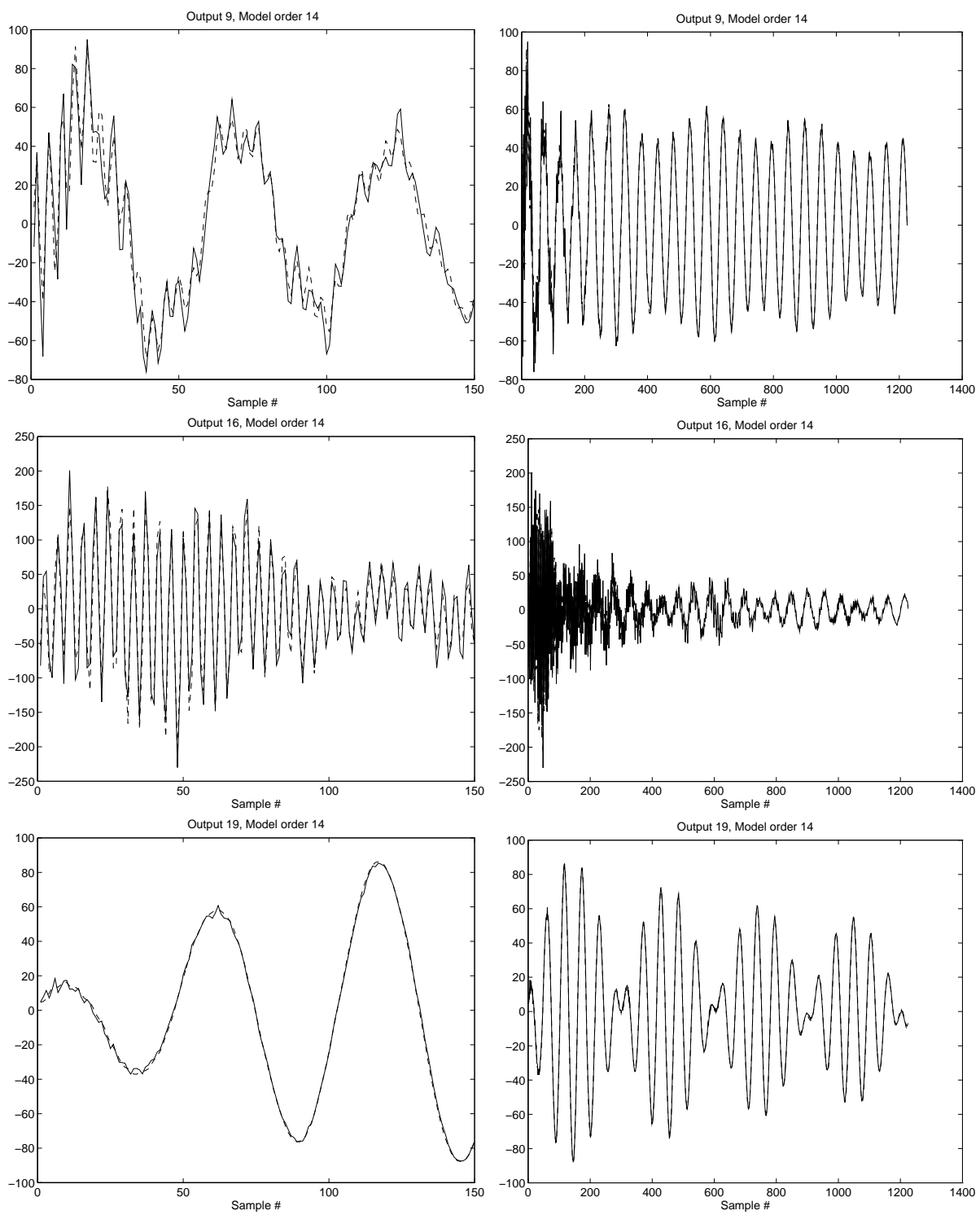

Figure 6: Selected measured (solid) and simulated (dashed) normalized accelerations. The simulated outputs originate from an estimated model of order 14. Top graphs show wing tip vertical acceleration. Middle graphs show nacelle lateral acceleration and bottom graphs show vertical acceleration of rear fuselage. The left graphs show the 150 first samples of the corresponding right graphs. 
initial model delivered by Kung's algorithm is unstable. The subsequent PEM iterations, however, yielded a stable model.

From the high Modal Coherence Indicators we can conclude that all identified frequencies are of high quality.

In Table 2 the average of the MCIs $\gamma_{k}$ are shown. We see from the average values that the prediction error minimization step improved the overall Modal Coherence. The PEM step has moved the frequencies only marginally but the damping ratios are more affected.

A comparison between the simulated impulse response from the estimated model and the measured signals are shown in Figure 6 for a selection of three accelerometer channels. The left graphs depict the initial 150 samples of the graphs shown to the right. All the graphs indicate that the estimated model is of high quality both for high frequency modes as well as the low frequency modes.

\subsection{Discussion}

The method described produced a state-space vibrational model which describes the measured accelerations with high accuracy. Even though the model is of considerable size (14 states and 21 outputs) the computational load is quite manageable. This stems from the fact that the realization algorithm produced an initial model of high quality and the particular parameterization used in the prediction error method. Moreover, the Modal Coherence quality measure and the global cross-validation, using independent output channels, provide significant aid, both when deciding model order and in the evaluation of the final model. As a result, the estimated modal frequencies and damping ratios can be used to further evaluate the vibrational properties of the aircraft for safety and comfort reasons.

\section{Conclusions}

We have found that the chosen identification method builds models that very accurately can reproduce the vibrational behavior of the aircraft. The initial, realization based, step gives a very good model, that is marginally, but clearly, further improved by a prediction error method. The vibrational modes of the resulting model are consistent with those obtained by other techniques. All in all, the methodology described here has been adopted by the SAAB Aircraft company for vibrational mode analysis and particularly

for flutter analysis, and has been found to be a time saving complement to conventional Fourier transform based techniques. 


\section{References}

[1] M. Basseville, A. Benveniste, B. Gach-Devauchelle, M. Goursat, D. Bonnecase, P. Dorey, M. Prevosto, and M. Olagnon. Damage monitoring in vibration mechanics: issues in diagnostics and predictive maintenance. Mechanical Systems and Signal Processing, 7(5):401-423, Sept. 1993.

[2] M. Basseville, A. Benveniste, G. Moustakides, and A. Rougée. Detection and diagnosis of changes in the eigenstructure of nonstationary multivariable systems. Automatica, 23(4):479-489, 1987.

[3] A. Benveniste and J. J. Fuchs. Single sample modal identification of a nonstationary stochastic process. IEEE Trans. of Automatic Control, 30(1):66-74, 1985.

[4] P. De Groen and B. De Moor. The fit of a sum of exponentials to noisy data. Journal of Computational and Applied Mathematics, 20:175-187, 1987.

[5] G. R. de Prony. Essai expérimental et analytic, etc. J. L'Ecole Polytechnique, Paris, 1(2):24-76, 1795.

[6] J. E. Dennis and R. B. Schnabel. Numerical Methods for Unconstrained Optimization and Nonlinear Equations. Prentice-Hall, Englewood Cliffs, New Jersey, 1983.

[7] F. B. Hildebrand. Introduction to Numerical Analysis. McGraw-Hill, New-York, 1956. ch. 9.

[8] B. L. Ho and R. E. Kalman. Effective construction of linear state-variable models from input/output functions. Regelungstechnik, 14(12):545-592, 1966.

[9] J. N. Juang and R. S. Pappa. An eigensystem realization algorithm for modal parameter identification and model reduction. J. of Guidance, Control and Dynamics, 8(5):620-627, 1985.

[10] S. M. Kay. Modern Spectral Estimation, Theory $\&$ Application. Prentice-Hall, Englewood Cliffs, New Jersey, 1988.

[11] L. Kronecker. Zur theorie der elimination einer variabeln aus zwei algebraischen gleichungen. Trans. Royal Prussian Academy of Science., 1881. see collected works Vol. 2.

[12] S. Y. Kung. A new identification and model reduction algorithm via singular value decomposition. In Proc. of 12th Asilomar Conference on Circuits, Systems and Computers, Pacific Grove, CA, pages 705-714, 1978. 
[13] C. Lanczos. Applied Analysis. Prentice Hall, Englewood Cliffs, NJ, 1956.

[14] J. S. Lew, J. N. Juang, and R. W. Longman. Comparison of several system identification methods for flexible structures. J. of Sound and Vibration, 167(3):461-480, 1993.

[15] L. Ljung. System Identification: Theory for the User. Prentice-Hall, Englewood Cliffs, New Jersey, 1987.

[16] T. McKelvey. On state-space models in system identification. Licentiate Thesis 447, Electrical Engineering, Linköping University, S-581 83 Linköping, Sweden, May 1994.

[17] M. Prevosto, M. Olagnon, A. Benveniste, and M. Basseville. State space formulation: A solution to modal parameter estimation. Journal of Sound and Vibration, 148(2):329-342, 1991.

[18] R. Roy, A. Paulray, and T. Kailath. ESPRIT-A subspace rotation approach to estimation of parameters of cisoids in noise. IEEE Trans. on Acoustics, Speech and Signal Processing, ASSP-34(5):1340-1342, October 1986 .

[19] L. M. Silverman. Realization of linear dynamical systems. IEEE Trans. on Automatic Control, 16(6):554-567, 1971.

[20] P. Stoica, R. Moses, B. Friedlander, and T. Söderström. Maximum likelihood estimation of the parameters of multiple sinusoids from noisy measurements. IEEE Trans. on Acoustics, Speech and Signal Processing, 37(3):378-392, March 1989.

[21] H. Vold and R. Russel. Advanced analysis methods improve modal test results. Sound and Vibration, pages 36-40, 1983.

[22] D. C. Youla and P. Tissi. n-port synthesis via reactance extraction Part I. IEEE Conv. Rec., 14(7):183-205, March 1966.

[23] H. P. Zeiger and A. J. McEwen. Approximate linear realizations of given dimension via Ho's algorithm. IEEE Trans. on Automatic Control, 19:153, April 1974. 